\title{
OS AVANÇOS DA TECNOLOGIA ASSISTIVA PARA PESSOAS COM PARALISIA CEREBRAL NO BRASIL: REVISÃO DE LITERATURA
}

\author{
Romilson Cesar LIMA ${ }^{1}$ \\ Jesse Bergamini FURLAN ${ }^{2}$ \\ Sandra Regina Gonçalves SANTOS ${ }^{3}$ \\ Rafael BARREROS ${ }^{4}$ \\ Edson Elidio ADÃO 5 \\ Regiane Luz CARVALHO ${ }^{6}$
}

\begin{abstract}
${ }^{1}$ Graduado em Administração de Empresas (UNIP), Especialista em Gestão Empresarial (PUC) e MBA em Gestão Industrial (FGV), Mestrando em Qualidade de Vida - UNIFAE. romilson_cesar@uol.com.br ${ }^{2}$ Graduado em Engenharia Metalúrgica-Modalidade Produção-FEI, Engenheiro de Segurança do Trabalho, Mestrando em Qualidade de Vida-UNIFAE; Professor da UNIFAE. furlan@ feralvarez.com.br ${ }^{3}$ Graduada Letras (UNIFEOB), Comunicação Social (UNIFAE) , Pós-Graduada em Educação Empreendedora (UFSJ) e Mestranda em Qualidade de Vida - (UNIFAE). Professora do SENAI. sandracomunicacao@gmail.com

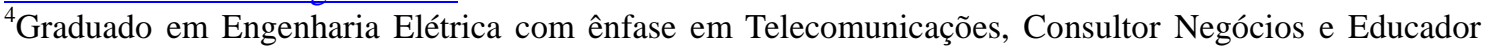
Financeiro. rbarreros@ yahoo.com.br

${ }^{5}$ Graduado em Educação Artística, Especialista em Psicopedagogia e Gestão Escolar, Mestrando em Qualidade de Vida - UNIFAE, Professor do IFSP. edson_elidio@bol.com.br

${ }^{6}$ Graduada em Fisioterapia, mestre e doutora em neurofisiologia UNICAMP, Pós doutora em bioengenharia USP, professora do mestrado em qualidade de vida UNIFAE. regianeluzcarvalho@gmail.com
\end{abstract}

\section{Recebido em: 28/05/2014 - Aprovado em: 20/09/2014 - Disponibilizado em: 15/12/2014}

\section{Resumo}

Paralisia Cerebral é uma desordem sensório-motora na qual os problemas vão além dos físicos e cognitivos. As dificuldades são verificadas em aspectos ambientais/arquitetônicos e atitudinais. Neste sentido a mobilização de vários segmentos da sociedade em função das demandas das pessoas com Paralisia Cerebral vem contribuindo para o desenvolvimento de Tecnologias Assistivas (TAs) que impulsionem o processo de uma sociedade mais justa e igualitária. Neste contexto esta revisão objetivou apresentar os avanços a respeito da TA para pessoas com Paralisia Cerebral. A pesquisa foi conduzida em bases de dados de acesso público no período entre 2006 e 2014. Foram encontrados 20 artigos sendo que 10 abordavam a TA para pessoas com Paralisia Cerebral. Foram avaliadas 105 pessoas sendo que o principal instrumento foi a entrevista semi-estruturada. Os resultados indicam os efeitos positivos da TA em todos os trabalhos. Embora os efeitos benéficos sejam notórios, dificuldades foram relatadas como: falta de treinamento para utilização correta da TA, ausência de participação da pessoa com deficiência na escolha do recurso a ser utilizado, falta de sistematização, dificuldade financeira e alto custo. Diante destas dificuldades é indispensável a ampliação e diversificação de recursos de TA e modelos de equipamentos oferecidos pelo SUS, de forma a contemplar todos os domínios da vida cotidiana de seus usuários assim como de programas de treinamento para os professores e campanha de divulgação.

Palavras-chave: Tecnologia Assistiva, paralisia cerebral, sociedade.

\begin{abstract}
:
Cerebral Palsy is a sensorimotor disorder in which the problems go beyond the physical and cognitive. The difficulties are checked in environmental/architectural and attitudinal aspects. In this sense the mobilization of various segments of society in the light of the demands of people with cerebral palsy has been contributing to the development of Assistive Technologies (TA) that drive the process of a more just and egalitarian society. In this context, this review aims to present the advances regarding TA for people with Cerebral Palsy. The research was conducted in databases accessible to the public between 2006 and 2014. 20 articles were found while 10 addressed the Assistive Technology for People with Cerebral Palsy.
\end{abstract}


105 people were evaluated and the main instrument was the semi - structured interview. The results indicate the positive effects of TA on all jobs. Although the beneficial effects are notorious difficulties were reported as lack of training for correct use of the TA, the absence of participation of people with disabilities in choosing the resource to be used, lack of systematization, financial difficulty and high cost. Faced with these difficulties is essential to expansion and diversification of resources and TA equipment models offered by SUS in order to cover all areas of everyday life of its users as well as training programs for teachers and outreach campaign.

Keywords: Assistive Technology, cerebral paralysis, society.

\section{Introdução}

Segundo a Organização Mundial da Saúde (OMS), 10\% da população mundial é composta de pessoas com algum tipo de deficiência. No Brasil, a estatística revela que $14,5 \%$ da população apresentam alguma deficiência atingindo em torno de 27 milhões de pessoas. Filho (2011) informa que grande parcela dessa população vive uma realidade de carência social, com baixa renda e baixo nível de escolarização, o que só potencializa suas dificuldades em função das barreiras, preconceitos, desigualdades e desinformação (FERREIRA, 2011).

Dentre as deficiências destaca-se a Paralisia Cerebral com cerca de 30 a 40 mil casos novos por ano (OLIVEIRA et al., 2011). Sua incidência estimada é de 1,5 a 2,5 para cada 1.000 nascidos vivos nos países desenvolvidos e de 7 para cada 1.000 nascidos vivos em países em desenvolvimento. É uma desordem sensório-motora que envolve distúrbios do tônus muscular, postura e movimentos voluntários, ocasionada por lesão cerebral, associada com hipóxia ou anóxia no período de maturação estrutural e funcional do cérebro.

A paralisia cerebral vai além dos problemas físicos e cognitivos. A criança portadora da doença, ao crescer, enfrenta dificuldades diárias para interagir com os colegas e frequentar escolas de educação regular, já que muitas não estão preparadas para recebê-las de uma forma inclusiva. As dificuldades são verificadas em dois aspectos: ambientais/arquitetônicos e atitudinais.

Neste sentido, a mobilização de vários segmentos $\mathrm{da}$ sociedade $\mathrm{em}$ função das demandas das pessoas com Paralisia Cerebral vem contribuindo para o desenvolvimento de tecnologias que impulsionem o processo de uma sociedade mais justa e igualitária. 
Gradativamente são feitos investimentos na direção de se produzir e aplicar conhecimentos em produtos específicos para essa população que vem recebendo o nome de Tecnologias Assistivas (TAs) (ALVES \& MATSUKURA, 2011).

Essas novas tecnologias vêm sendo incorporadas em nossa cultura, caracterizando-se cada vez mais como ferramenta indispensável na inclusão e integração de pessoas com algum tipo de deficiência (ROCHA, 2010). A constatação é ainda mais evidente e verdadeira quando se refere às pessoas com dificuldades na comunicação (oral e escrita), na funcionalidade $\mathrm{e}$ locomoção (OLIVEIRA, GAROTTI e SA, 2008).

Os avanços das TAs contribuem para proporcionar maior habilidade e autonomia na realização das atividades das pessoas com deficiência, promovendo a inclusão social e a independência, proporcionando prérequisitos para leitura, escrita e inserção social. (PELOSI et al., 2011).

Apesar dos grandes avanços no que diz a respeito à inclusão das pessoas com deficiência com auxílio da TA, existe uma lacuna entre os direitos e a efetivação do acesso. Esta lacuna motivou os autores deste trabalho a realizarem um levantamento bibliográfico com o propósito de mapear a situação de pessoas com Paralisia Cerebral no ambiente familiar, escolar e em atividades externas e compreender os fatores diferenciais que podem facilitar ou dificultar o uso das TAs.

\section{Metodologia}

Este estudo consistiu em uma revisão da literatura, sem metanalise, sobre as possíveis Tecnologias Assistivas utilizadas por pessoas com Paralisia Cerebral.

Estratégia de pesquisa

A pesquisa foi realizada nas bases de dados eletrônicas, nacionais e Internacionais, LILACS , MEDLINE , PED ro, PubMed e SciELO, através da consulta pelos seguintes descritores: Tecnologia Assistiva, Paralisia Cerebral, Acessibilidade junto de suas combinações, no período de 2006 a 2014.

Os artigos identificados pela estratégia de busca foram avaliados, obedecendo rigorosamente aos critérios de inclusão: texto na integra, tempo de busca, população-alvo (indivíduos com Paralisia Cerebral), tipo de estudo (sem 
delimitação) e idioma (português, inglês

e espanhol). Tais estratégias foram

tomadas com o intuito de maximizar os

resultados da pesquisa, uma vez que foi

constatada escassez de literatura.

Foram excluídos os estudos que

não obedeceram aos critérios de

inclusão supracitados e os estudos

desenvolvidos em outros países, visto

que o objetivo deste foi analisar o desenvolvimento das TAs para pessoas com Paralisia Cerebral no Brasil.

\section{Resultados}

Foram encontrados 29 artigos no período de 2006 a 2014, sendo que 10 abordavam os avanços das TAs para crianças com Paralisia Cerebral no Brasil e foram incluídos no estudo. Os dados foram organizados em uma tabela para posterior análise (Tabela1)

Tabela 1. Artigos selecionados sobre Tecnologia Assistiva

\begin{tabular}{|c|c|c|c|}
\hline Autor/Título & Objetivos & Metodologia/Participantes & hclusão \\
\hline $\begin{array}{lr}\text { OKIDOET } & \text { et } \text { al. } \\
\text { Revista da } & \text { Escola } \\
\text { de Enfermagem da } \\
\text { USP, } & \text { vol.46 } \\
\text { número } 5 & \text { São } \\
\text { Paulo } & 2012 . \\
\text { Criança } & \\
\text { Dependente } & \text { de } \\
\text { Tecnologia: } & \text { a } \\
\text { experiência } & \text { do } \\
\text { cuidado materno }\end{array}$ & $\begin{array}{l}\text { Compreender a } \\
\text { experiência } \\
\text { materna no cuidado } \\
\text { ao filho dependente } \\
\text { de TA. }\end{array}$ & $\begin{array}{l}\text { Criança de } 7 \text { anos com } \\
\text { diagnóstico de encefalopatia } \\
\text { crônica } 4 \text {-Análise de } \\
\text { prontuário e de tipo de } \\
\text { dependência tecnológica, } \\
\text { duração da dependência, no } \\
\text { de dispositivos de TA e } \\
\text { serviços onde fez } \\
\text { acompanhamento. }\end{array}$ & $\begin{array}{l}\text { A enfermagem apresentou um } \\
\text { papel fundamental no cuidado } \\
\text { às crianças dependentes de } \\
\text { tecnologia e suas famílias, } \\
\text { tendo o compromisso de } \\
\text { ampliá-las no processo de } \\
\text { transição para o domicílio.É } \\
\text { preciso avançar na busca por } \\
\text { uma assistência integral, } \\
\text { pautada na cooperação e na co- } \\
\text { responsabilidade. }\end{array}$ \\
\hline $\begin{array}{l}\text { Varela \& Oliver. } \\
\text { Revista Ciência \& } \\
\text { Saúde Coletiva } \\
\text { vol. 18 número 6 } \\
\text { Rio de janeiro Jun. } \\
\text { 2013. A utilização } \\
\text { de Tecnologia } \\
\text { Assistiva na vida } \\
\text { cotidiana } \\
\text { crianças de } \\
\text { deficiência. }\end{array}$ & $\begin{array}{l}\text { Compreender a } \\
\text { utilização da TA na } \\
\text { vida cotidiana por } \\
\text { crianças com } \\
\text { deficiência. }\end{array}$ & $\begin{array}{l}\text { Participaram } 5 \text { crianças com } \\
\text { Paralisia Cerebral e idade } \\
\text { entre } 5 \text { e } 14 \text { anos. Foram } \\
\text { observadas durante atividades } \\
\text { que realizavam em casa e na } \\
\text { escola. Foram realizadas } \\
\text { entrevistas com os } \\
\text { cuidadores sobre a percepção } \\
\text { sobre as atividades diárias, a } \\
\text { deficiência, e a TA. }\end{array}$ & $\begin{array}{l}\text { A TA ofereceu a criança } \\
\text { oportunidade } \\
\text { amadurecimento, } \\
\text { introdução na convívio, } \\
\text { participação e inclusão social. } \\
\text { No entanto, não garantiu a } \\
\text { facilitação da vida cotidiana. }\end{array}$ \\
\hline $\begin{array}{lr}\text { ALCASSA, et } & \text { al. } \\
2013 . \quad \text { Crianças } \\
\text { Tetraparéticas } & \text { e } \\
\text { cuidadores: } & \\
\text { caracterizando } & \text { o } \\
\text { perfil e } & \text { a } \\
\text { acessibilidade } & \text { à } \\
\text { Tecnologia } & \\
\text { Assistiva. } & \end{array}$ & $\begin{array}{l}\text { Descrever o perfil } \\
\text { dos cuidadores e } \\
\text { das crianças com } \\
\text { tetraparesia } \\
\text { segundo suas } \\
\text { características, } \\
\text { aspectos } \\
\text { socioedemográficos } \\
\text { e acessibilidade }\end{array}$ & 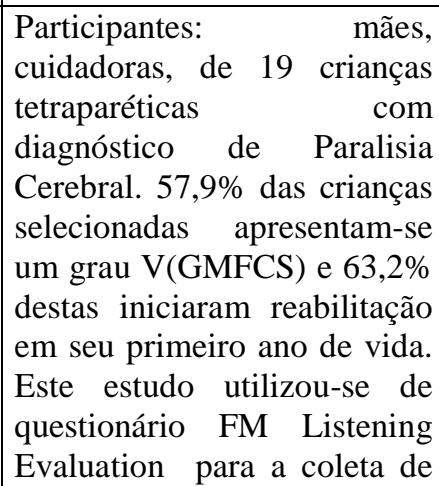 & $\begin{array}{l}68,4 \% \text { dos entrevistados } \\
\text { relataram a falta de recurso } \\
\text { financeiro como o principal } \\
\text { motivo para a não utilização de } \\
\text { TA. Isto reforça a necessidade } \\
\text { de maior investimento nesta } \\
\text { área, tanto do ponto de vista do } \\
\text { desenvolvimento de TA no } \\
\text { Brasil, como também em } \\
\text { políticas públicas que facilitem } \\
\text { o acesso a estas tecnologias. }\end{array}$ \\
\hline
\end{tabular}




\begin{tabular}{|c|c|c|c|}
\hline & & $\begin{array}{l}\text { dados, foi composto de } \\
\text { questões que abordaram o } \\
\text { conhecimento sobre as } \\
\text { características das crianças , } \\
\text { aspectos sociodemográficos, } \\
\text { acessibilidade e Tecnologias } \\
\text { Assistivas. }\end{array}$ & \\
\hline $\begin{array}{lr}\text { Rocha } & \& \\
\text { Deliberato. } & \\
\text { Tecnologia } & \\
\text { Assistiva para a } \\
\text { criança } r \text { com } \\
\text { paralisia cerebral } \\
\text { na } & \text { escola: } \\
\text { identificação das } \\
\text { necessidades. }\end{array}$ & $\begin{array}{l}\text { Identificar } \text { a } \\
\text { necessidade de } \\
\text { serviços, recursos e } \\
\text { estratégias de TA } \\
\text { para o aluno com } \\
\text { paralisia cerebral } \\
\text { na escola. }\end{array}$ & $\begin{array}{l}\text { Participaram } 2 \text { crianças com } \\
3 \text { e } 6 \text { anos e diagnóstico } \\
\text { médico de paralisia cerebral. } \\
\text { Procedimentos: entrevista } \\
\text { com os professores utilizando } \\
\text { roteiro semiestruturado, } \\
\text { preenchimento pelo professor } \\
\text { do protocolo de identificação } \\
\text { da rotina escolar, observação } \\
\text { dos participantes em sala de } \\
\text { aula através de filmagens e } \\
\text { registro contínuo em diário de } \\
\text { campo. }\end{array}$ & $\begin{array}{l}\text { Os autores apontam que a } \\
\text { caracterização dos alunos e do } \\
\text { ambiente é fundamental para a } \\
\text { confecção dos recursos da TA e } \\
\text { a importância da capacitação do } \\
\text { professor não só para uso dos } \\
\text { recursos, mas para estabelecer } \\
\text { as estratégias de uso dos } \\
\text { materiais. Apontam também o } \\
\text { desconhecimento dos } \\
\text { cuidadores sobre o termo TA. }\end{array}$ \\
\hline $\begin{array}{lr}\text { Alves } & \& \\
\text { Matsukura. } & \\
\text { Percepção } & \text { de } \\
\text { Alunos } & \text { com } \\
\text { paralisia } & \text { cerebral } \\
\text { sobre o uso de } & \text { recursos } \\
\text { tecnologia } & \text { de } \\
\text { assistiva na } & \\
\text { regular. } & \end{array}$ & $\begin{array}{l}\text { Buscou investigar } \\
\text { os efeitos do uso da } \\
\text { TA no contexto da } \\
\text { escolarização do } \\
\text { aluno com paralisia } \\
\text { cerebral a partir de } \\
\text { sua própria } \\
\text { percepção, ra } \\
\text { percepção do seu } \\
\text { professor e de seu } \\
\text { cuidador. }\end{array}$ & $\begin{array}{l}\text { Dois alunos com nível motor } \\
\text { IV, três alunos com nível } \\
\text { motor V, e idade entre oito a } \\
\text { doze anos diagnosticados } \\
\text { com paralisia cerebral que } \\
\text { faziam uso, há pelo menos } \\
\text { seis meses, de recurso de TA } \\
\text { na execução de tarefas de } \\
\text { escrita e/ou comunicação. } \\
\text { Métodos:1- } \\
\text { semiestruturado dividido em } \\
4 \text { temas: a)-TA b)-Tarefas de } \\
\text { sala de aula c)-Socialização } \\
\text { d)-Inclusão Social. }\end{array}$ & $\begin{array}{l}\text { Nenhuma criança participou da } \\
\text { escolha de seu recurso de TA. } \\
\text { Apontam que os recursos de TA } \\
\text { auxiliam na execução de tarefas } \\
\text { e participação nas atividades em } \\
\text { classe e que trouxeram } \\
\text { contribuições ao processo de } \\
\text { escolarização. Os autores } \\
\text { indicam uma necessidade de } \\
\text { sistematização } \\
\text { acompanhamento } \\
\text { utilização de TA. }\end{array}$ \\
\hline $\begin{array}{l}\text { COSTA, et al } \\
2009 \text {. Cadeira } \\
\text { especial para o } \\
\text { estudo } \\
\text { videofluoroscópico } \\
\text { da deglutição e } \\
\text { suas disfunções }\end{array}$ & $\begin{array}{l}\text { Configurar } \\
\text { equipamento que } \\
\text { permita avaliação } \\
\text { videofluoroscópica } \\
\text { da deglutição e } \\
\text { suas desordens sem } \\
\text { a necessidade de } \\
\text { colaboração dos } \\
\text { indivíduos na } \\
\text { obtenção r das } \\
\text { posições requeridas } \\
\text { pelas deficiências } \\
\text { associadas. }\end{array}$ & $\begin{array}{l}\text { Participantes: } 5 \text { crianças com } \\
\text { menos de } 5 \text { anos de idade. } \\
\text { Método: Utilizaram como } \\
\text { estrutura básica um arco e } \\
\text { uma cadeira especial com a } \\
\text { finalidade de permitir que os } \\
\text { exames videofluoroscópicos } \\
\text { das três fases da deglutição } \\
\text { (oral, faríngea e esofágica). }\end{array}$ & $\begin{array}{l}\text { A cadeira possibilitou maior } \\
\text { efetividade qualitativa, por } \\
\text { permitir de modo não invasivo a } \\
\text { adequadarane } \\
\text { morfofuncional das três fases da } \\
\text { deglutição. }\end{array}$ \\
\hline
\end{tabular}




\begin{tabular}{|c|c|c|c|}
\hline \begin{tabular}{|l} 
OLIVEIRA, et al \\
2013 . \\
desenvolvimento \\
da roupa \\
biocinética
\end{tabular} & $\begin{array}{l}\text { Promover o } \\
\text { desenvolvimento } \\
\text { de dispositivos de } \\
\text { tecnologia assistiva } \\
\text { economicamente } \\
\text { acessíveis. }\end{array}$ & $\begin{array}{l}\text { Foram construídos cinco } \\
\text { protótipos da roupa } \\
\text { biocinética, de diferentes } \\
\text { modelos, } \\
\text { posicionamento das presilhas } \\
\text { e tiras elásticas. Os protótipos } \\
\text { foram desenvolvimentos } \\
\text { considerando r as } \\
\text { características neuromotoras } \\
\text { de duas crianças. As roupas } \\
\text { foram experimentadas e } \\
\text { ajustadas. A fase de teste da } \\
\text { eficácia da roupa ainda será } \\
\text { iniciada. Os protótipos foram } \\
\text { evoluindo na medida em que } \\
\text { se observaram seus pontos } \\
\text { positivos ou negativos } \\
\text { durante a experimentação. }\end{array}$ & $\begin{array}{l}\text { Percebeu-se que tais protótipos } \\
\text { possibilitaram maior suporte } \\
\text { corporal, o favorecimento da } \\
\text { estabilidade e, ainda, } \\
\text { proporcionaram input de } \\
\text { estímulos proprioceptivos e a } \\
\text { inibição de padrões patológicos } \\
\text { pertinentes à deficiência } \\
\text { neuromotora. }\end{array}$ \\
\hline $\begin{array}{|lrr|}\begin{array}{l}\text { PELOSI, } \\
2011 . \quad \text { A t }\end{array} & \text { ação } \\
\text { conjunta } & \text { dos } \\
\text { profissionais } & \text { da } \\
\text { saúde } & \text { e } & \text { da } \\
\text { educação na } & \text { escola } \\
\text { inclusiva. } & \end{array}$ & $\begin{array}{l}\text { O objetivo do } \\
\text { estudo foi avaliar } \\
\text { se a introdução da } \\
\text { Tecnologia } \\
\text { Assistiva favorece } \\
\text { a inclusão de } \\
\text { alunos r com } \\
\text { paralisia cerebral } \\
\text { em quatro escolas } \\
\text { regulares r do } \\
\text { município do Rio } \\
\text { de Janeiro quando } \\
\text { esta é mediada pela } \\
\text { ação conjunta de } \\
\text { profissionais da } \\
\text { Saúde e Educação. }\end{array}$ & $\begin{array}{l}\text { Participaram do estudo } \\
\text { quatorze professores, cinco } \\
\text { terapeutas ocupacionais, três } \\
\text { fonoaudiólogas, quatro } \\
\text { diretores, quatro alunos com } \\
\text { paralisia cerebral e seus } \\
\text { familiares, quatro professores } \\
\text { de turma e cinco professores } \\
\text { de aulas complementares. } \\
\text { Foram risados } \\
\text { questionários, entrevistas } \\
\text { semiestruturadas, registros } \\
\text { dos participantes, fotografias, } \\
\text { filmes e o caderno de campo } \\
\text { da pesquisadora. }\end{array}$ & $\begin{array}{l}\text { O emprego sistemático de } \\
\text { recursos de TA teve efeito } \\
\text { positivo na postura dos alunos } \\
\text { em sala de aula, no } \\
\text { desenvolvimento de estratégias } \\
\text { alternativas de comunicação } \\
\text { oral e escrita, no aumento da } \\
\text { mobilidade dos alunos através } \\
\text { do uso de cadeiras de rodas } \\
\text { apropriadas e na independência } \\
\text { para as atividades do dia-a-dia } \\
\text { escolar. Os resultados da ação } \\
\text { conjunta compreenderam } \\
\text { situações de aprendizagem } \\
\text { favoráveis à inclusão escolar; } \\
\text { sensibilização e mudança de } \\
\text { atitude dos professores, e } \\
\text { aproximação de professores e } \\
\text { alunos. }\end{array}$ \\
\hline 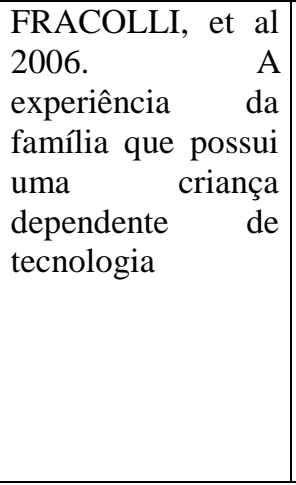 & $\begin{array}{l}\text { O objetivo do } \\
\text { estudo } \\
\text { compreender foi } \\
\text { experiência } \\
\text { das famílias que } \\
\text { possuem crianças } \\
\text { dependentes de TA }\end{array}$ & $\begin{array}{l}\text { Participaram do estudo sete } \\
\text { famílias, com crianças de } \\
\text { idade variando entre } 9 \text { meses } \\
\text { e } 14 \text { anos. As mães foram } \\
\text { avaliadas por entrevista } \\
\text { semiestruturada. }\end{array}$ & $\begin{array}{l}\text { A realidade destas famílias em } \\
\text { nosso país e em seus diferentes } \\
\text { cenários socioeconômicos } \\
\text { necessita ser mais amplamente } \\
\text { pesquisada. O processo de } \\
\text { trabalho com famílias de } \\
\text { crianças dependentes de } \\
\text { configura-se como uma } \\
\text { modalidade complexa de } \\
\text { intervenção em saúde } \\
\text { necessita ser embasado em } \\
\text { resultados de pesquisa. }\end{array}$ \\
\hline
\end{tabular}




\begin{tabular}{|c|c|c|c|}
\hline $\begin{array}{l}\text { OLIVEIRA, et al, } \\
\text { 2008. A tecnologia } \\
\text { e o } \\
\text { desenvolvimento } \\
\text { cognitivo da } \\
\text { criança com } \\
\text { paralisia cerebral }\end{array}$ & $\begin{array}{l}\text { Abordar o } \\
\text { desenvolvimento } \\
\text { da criança com } \\
\text { sequela de paralisia } \\
\text { cerebral, como } \\
\text { recurso pedagógico } \\
\text { de possibilidades } \\
\text { em sala de aula e a } \\
\text { analise da sua inter- } \\
\text { relação e } \\
\text { interdependência } \\
\text { com a comunicação } \\
\text { alternativa, } \\
\text { suplementar e/ ou } \\
\text { aumentativa. }\end{array}$ & $\begin{array}{l}\text { Participaram } 5 \text { crianças } \\
\text { quadriplégicas na faixa etária } \\
\text { de } 05 \text { a } 07 \text { anos. Foram } \\
\text { realizadas entrevistas } \\
\text { informais com os pais para } \\
\text { levantar possíveis causas da } \\
\text { paralisia, início, tipo de } \\
\text { tratamento, desenvolvimento } \\
\text { neuropsicomotor, contexto } \\
\text { familiar, recursos utilizados } \\
\text { pela criança e tipo de escola. }\end{array}$ & 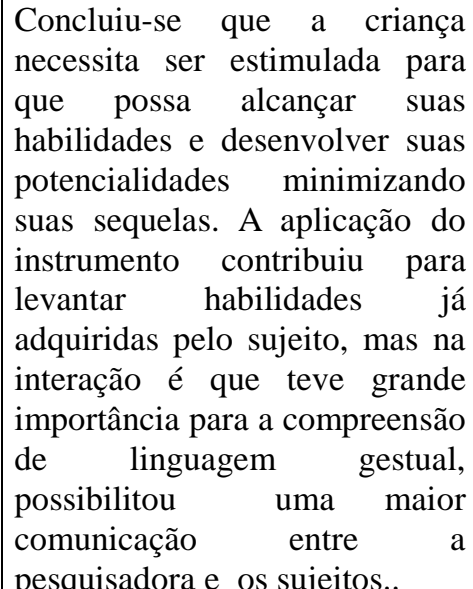 \\
\hline
\end{tabular}

\section{Discussão}

Os estudos abordados avaliaram um total de 105 pessoas com idade entre 9 messes e 14 anos. A metodologia de avaliação mais utilizada foi a entrevista semi estruturada (oito artigos) seguida de observação (sete artigos), protocolo de registros (quatro artigos) e aplicação de questionários para análise da rotina em casa e/ ou na escola (dois artigos).

A percepção da família em relação ao uso das TAs foi avaliada em $30 \%$ dos artigos. Destes, todos apontaram o efeito positivo dos recursos utilizados corroborando com os achados de Péres (2013) que atribui como principal objetivo das TAs o de proporcionar à pessoa com deficiência maior independência, qualidade de vida e inclusão social através da ampliação de sua comunicação, mobilidade, controle de seu ambiente, habilidades de seu aprendizado e trabalho.

Embora os efeitos benéficos sejam notórios, dificuldades foram relatadas como: falta de treinamento para utilização correta da TA, ausência de participação da pessoa com deficiência na escolha do recurso, falta de sistematização, dificuldade financeira e alto custo.

Estes aspectos foram abordados também em outros estudos que indicam a necessidade de participação do usuário de TAs no processo de escolha e implementação do recurso, assim como a realização de treinamento para o bom desempenho do usuário e adesão ao recurso (SCHERER et al., 2005; RIEMER-REISS; ＷACKER， 2000; COPLEY; ZIVIANI, 2004). Hipotetizase que esse fator possa influenciar negativamente a motivação, empenho 
do usuário e, consequentemente, fazer com que o recurso torne-se descontextualizado e perca seu propósito.

Em relação ao alto custo, Varela e Oliver (2013) destacam que no Brasil, muitos recursos desenvolvidos nas Universidades não são incorporados ao mercado. A variedade de produtos fabricados no país é pequena e grande parte dos dispositivos importados não dispõe de isenção tributária, elevando significativamente o custo das TAs disponíveis no mercado. Aliado a este fato associa-se os dados de que crianças com Paralisia Cerebral de nível socioeconômico baixo apresentaram desempenho inferior nas habilidades de autocuidado e mobilidade em comparação à crianças de nível socioeconômico maior (Madeira et al 2013).

Como pode ser observado o fator financeiro foi a principal causa da não utilização das TAs. Em relação a este aspecto Oliveira (2013) aponta a possibilidade de desenvolvimento de tecnologias de baixo custo no Brasil como uma saída para popularização das TAs.

Outro aspecto relevante apontado na literatura foi a utilização das TAs nas escolas. A percepção da família, dos professores e das próprias crianças foi avaliada em $40 \%$ dos artigos. Os resultados indicaram 0 desconhecimento dos professores sobre as TAs, a falta de avaliação do ambiente escolar para prescrição adequada das TAs assim como a falta de treinamento para os professores.

O período escolar é caracterizado como uma importante fase para o desempenho ocupacional da criança. Nesta fase não só o contexto físico e social se alarga e diferencia, mas também as expectativas do meio social se tornam mais exigentes. A dependência é menos tolerada, as regras implícitas de convivência ficam mais complexas e o suporte está menos disponível. A exposição ao julgamento é mais evidente e instiga a criança a corresponder às expectativas da família, do professor e dos companheiros (MARTURANO, 2004).

Portanto, segundo ERIC (1993) os professores devem ter conhecimento e habilidades necessárias para selecionar e adaptar o currículo e o método de ensino de acordo com as necessidades individuais dos estudantes. Esta preparação não deve se restringir aos métodos e recursos especializados, mas 
sim propiciar orientação que leve ao desenvolvimento de competências e habilidades, que permitam a exploração e o domínio cada vez mais amplo das práticas docentes (MANTOAN, 1997).

A falta de preparo profissional dos docentes e a ausência de suporte por parte de órgãos competentes são fatos já corroborados por várias pesquisas (ERIC, 2002), inclusive esta. Não obstante, somente isto não pode ser usado como justificativa para a ausência de uma sociedade inclusiva no país. Para tanto e de acordo com Copley e Ziviani (2004), há a necessidade de ampliar e diversificar os recursos de TA e modelos de equipamentos oferecidos pelo SUS, de forma a contemplar todos os domínios da vida cotidiana de seus usuários.

\section{Conclusão}

A dificuldade de se encontrar informações a respeito das TAs indica que existe ainda uma grande lacuna entre as diretrizes legais existentes e a efetivação do usos desses recursos.

A TA se mostrou importante na facilitação do desenvolvimento das crianças com Paralisa Cerebral. Por meio das TAs a pessoa pode vivenciar o mundo que a cerca, minimizando os efeitos das barreiras motoras, interagindo e construindo conhecimentos e habilidades, favorecendo sua inclusão social e melhorando sua qualidade de vida.

Existem ainda dificuldades relacionadas à implementação das TAs que devem ser superadas como por exemplo o alto custo, a falta de conhecimento dos familiares e dos professores e a pouca participação da pessoa com deficiência na escolha do recurso a ser utilizado.

\section{Referências Bibliográficas}

ALVES, Ana Cristina de Jesus. Percepção de Alunos com Paralisia Cerebral sobre o uso de recursos de tecnologia assitiva na escola regular. Rev. Bras, Ed Esp., Marília, v 17, n 2 p 287-304, Maio- Ago. 2011

BERTOLUZZI, Renata Cristina. Autilização de Tecnologia Assistiva na vida cotidiana de crianças com deficiência. Revista Ciência \& Saúde Coletiva vol. 18 número 6. Rio de Janeiro, Jun 2013.

COPLEY, J., Ziviani J. Barriers to the use of assistive technology for children with multiple disabilities. Occup Ther Intern 2004; 11(4):229-243. 
COSTA, M.M.B. et al. Cadeira especial para o estudo videofluoroscópico da deglustição e suas disfunções. Radiologia Brasileira, v.42, n.3, São Paulo, mai./jun. 2009. http://dx.doi.org/10.1590/S0100$\underline{3984200900030001}$

ERIC, D. e BLAYA A.C. Violência nas escolas e políticas públicas. Brasília 2002: 268 p. UNESCO, 2002.

FILHO, G. T. Favorecendo práticas pedagógicas inclusivas por meio da Tecnologia Assistiva. In: NUNES, L. R. O. P.; PELOSI, M. B.; WALTER, C. C. F. (orgs.). Compartilhando experiências: ampliando a comunicação alternativa. Marília: ABPEE, p. 71-82, 2011.

FRACOLLI, R.A. et al. A experiência da família que possui criança dependente de tecnologia. Revista Mineira de Enfermagem, v.10, n.2, Minas Gerais, abr./jun. 2006.

GALVÃO, Cláudia Regina Cabral. Crianças tetraplégicas e a cuidadora caracterizando o perfil e a acessibilidade e à Tecnologia Assistiva., ISSN 0104-4931 Cad. Ter. Ocup. UFSCar, São Carlos, v. 21, n. 1, p. 1118 , 2013 http://dx.doi.org/10.4322/cto.2013.003
GALVÃO FILHO, T. A. A Tecnologia Assistiva: de que se trata? In: MACHADO, G. J. C.; SOBRAL, M. N. (Orgs.). Conexões: educação, comunicação, inclusão e interculturalidade. 1 ed. Porto Alegre: Redes Editora, p. 207-235, 2009. (disponível no formato PDF em < www.galvaofilho.net/assistiva.pdf $>$ ).

FERREIRA, Maria Inês Jesus Tecnologia Assistiva para crianças com paralisia cerebral sem oralidade: avaliação da comunicação durante atividades com jogos digitais - 2011 .

MADEIRA, A. et al. Desempenho funcional de crianças com paralisia cerebral de níveis socioeconômicos alto e baixo. Revista Paulista de Pediatria, 31(1):51-57, mar. 2013.

MANTOAN, Maria Tereza Egler. (Org.). 1997. A integração de pessoas com deficiência. São Paulo: Memnon. SENAC.

MARTURANO, E.M. (2004). Fatores de risco e proteção no desenvolvimento sócio-emocional de crianças com dificuldades de aprendizagem. Em E.G. Mendes, M.A.Almeida \&L.C.A. Williams (ORGs.). Avanços recentes em Educação Especial (pp 159-165). São Carlos: EDUFSCar. 
OLIVEIRA, A.I.A. et al. O desenvolvimento de roupa biocinética. Caderno de Terapia Ocupacional UFSCar, São Carlos, v.21, n.1, p.3-19, 2013.

http://dx.doi.org/10.4322/cto.2013.002

OLIVEIRA, A.I.A; GAROTTI, M.F.; SÁ, N.M.C.M. Tecnologia de ensino e tecnologia assistiva no ensino de crianças com paralisia cerebral. Revista Ciências \& Cognição. Vol. 13, 2008.

OLIVEIRA, Pinto, Ruffeil. A tecnologia e o desenvolvimento cognitivo da criança com paralisia cerebral. Ciências \& Cognição 2008: Vol. 13 (3):243_262. Disponível em: 〈htpp://cienciasecognicao@ ciencias>.

OKIDO, Ana Cristiane Cavicchioli. Criança Dependente de Tecnologia: a experiência do cuidado materno. Revista Escola de Enfermagem da USP, vol. 46 número 5 São Paulo. Outubro de 2012.

Organização Mundial da Saúde (OMS). Relatório mundial sobre a deficiência / World Health Organization, The World Bank ; tradução Lexicus Serviços Lingüísticos. - São Paulo, 2011.
PÉRES, D. D. Q. Tecnologias Assistivas como facilitadoras da aprendizagem significativa de crianças com deficiências. Gestão contemporânea, v.3, n.1, 2013.

PELOSI, M.B. ET AL. A ação conjunta dos profissionais da saúde e da educação na escola inclusiva. Revista de Terapia Ocupacional da Universidade de São Paulo, v.22, n.1, p.52-59, jan./abr. 2011.

PEREIRA, et al. Acessibilidade e crianças com paralisia cerebral: a visão do cuidador primário. 2011. Portaria $\mathrm{n}^{\circ}$ 142, de 16 de novembro de 2006 Diário Oficial da União, de $17 \mathrm{de}$ novembro de 2006.

ROCHA, Aila Narene Dahwache Criado. Processo de prescrição e confecção de recursos de tecnologia assistiva para educação infantil. 2010.

SASSAKI, R. K. 1996. Por que o termo “Tecnologia Assistiva"? Disponível em <http://www.assistiva.com.br/>. Acesso em 22 nov. 2007.

SCHERER, A. Estrutura trófica da avifauna em oito parques da cidade de Porto Alegre, Rio Grande do Sul, Ornithologia, 1(1): 25-32. 2005. 